\title{
Post-nephrectomy foreign-body granuloma in the retroperitoneum mimicking lymph node metastasis of renal cell cancer
}

This article was published in the following Dove Press journal:

OncoTargets and Therapy

20 November 2014

Number of times this article has been viewed

\author{
Jindan Luol,* \\ Yeqing Mao',* \\ Songliang Cai' \\ Xiaoyong Shen ${ }^{2}$ \\ Shanwen Chen' \\ Liping Xie'
}

'Department of Urology, The First Affiliated Hospital of College of Medicine, Zhejiang University,

Zhejiang, People's Republic of China;

${ }^{2}$ Department of Radiology, The

First Affiliated Hospital of College

of Medicine, Zhejiang University,

Zhejiang, People's Republic of China

*These authors contributed equally to this work
Correspondence: Shanwen Chen; Liping Xie

Department of Urology, The First Affiliated Hospital of College of Medicine, Zhejiang University, No 79 Qingchun

Road, Hangzhou, Zhejiang 310003 ,

People's Republic of China

Tel $+8657 \mid 87236832$

Fax +86 57। 87072577

Email zychenshanwen@।63.com;

xielp_zjuem@126.com

\begin{abstract}
Recently, iatrogenic foreign-body granuloma has been increasingly reported. The asymptomatic presentation and confusing appearance of granuloma can lead to misdiagnosis of a secondary malignancy, especially for a patient with a corresponding past medical history. Sometimes, surgical treatment is unavoidable, and the diagnosis relies upon the pathologic result. Herein, we report an unusual case of a 43-year-old man who underwent a nephrectomy for renal cell carcinoma (clear cell type) 5 years ago. A secondary granuloma was identified behind the inferior vena cava in the retroperitoneum 6 months after the surgery, but the radiologists had failed to identify it throughout the 4 years of routine examination. Later on, the lesion was identified by positron emission tomography, which classified it as a highly $18 \mathrm{~F}$-fluorodeoxyglucose-avid lesion. Considering no visible foreign-bodies identified on images, the lesion was arguably diagnosed as a lymph node metastasis of renal cancer. Finally, it was confirmed as a foreign-body granuloma encasing surgical suture and adipose tissue by the pathological analysis.
\end{abstract}

Keywords: iatrogenic foreign-body granuloma, positron emission tomography, 18F-fluorodeoxyglucose, renal cell carcinoma

\section{Introduction}

The retroperitoneal mass including a long list of various malignancies and benign tumors poses a challenge for differential diagnosis. Among these lesions, foreign-body granuloma, a kind of cancer-mimicking benign lesion, should be considered, especially when the patient presents with a past surgical history. The tumor-like, foreign-body granuloma results from a chronic inflammatory process in response to exogenous or endogenous deleterious agents.

Hitherto, increasing events of re-surgery due to unidentified granulomatous lesions in sites such as the breast, lung, thyroid, esophagus, extremity, and kidney have been reported; only a few of them have described misidentified granulomas in the retroperitoneal space. ${ }^{1-3}$ Here, we present a rare case of retroperitoneal foreign-body granuloma with a 5-year postoperative history of nephrectomy. The granuloma was diagnosed with lymph node metastasis of clear cell renal cell carcinoma (RCC) by both abdominal transverse computerized tomography (CT) and positron emission tomography (PET). The definitive diagnosis was not confirmed until the lesion was surgically resected and subjected to pathological analysis.

\section{Case report}

A 43-year-old man was diagnosed with a renal incidentaloma during a routine physical exam. No complications such as flank pain, fever, hematuria, or emaciation 
were presented. Physical examination revealed no other significant findings. Routine laboratory test results were unremarkable. The abdominal CT scan identified a welldefined, spheroidal mass with soft-tissue density located in the middle of the right kidney (Figure 1); the tumor presented with transient enhancement without visible visceral or lymph node involvement. The preoperative diagnosis was renal cancer. A laparoscopic radical nephrectomy was performed, and the pathologic exam revealed a clear cell RCC with moderate differentiation (Fuhrman II grade); no lymph node invasion was identified.

After discharge, the patient underwent regular exams in our clinic. Interestingly, the postoperative CT images were invariably misread as unremarkable by the radiologists
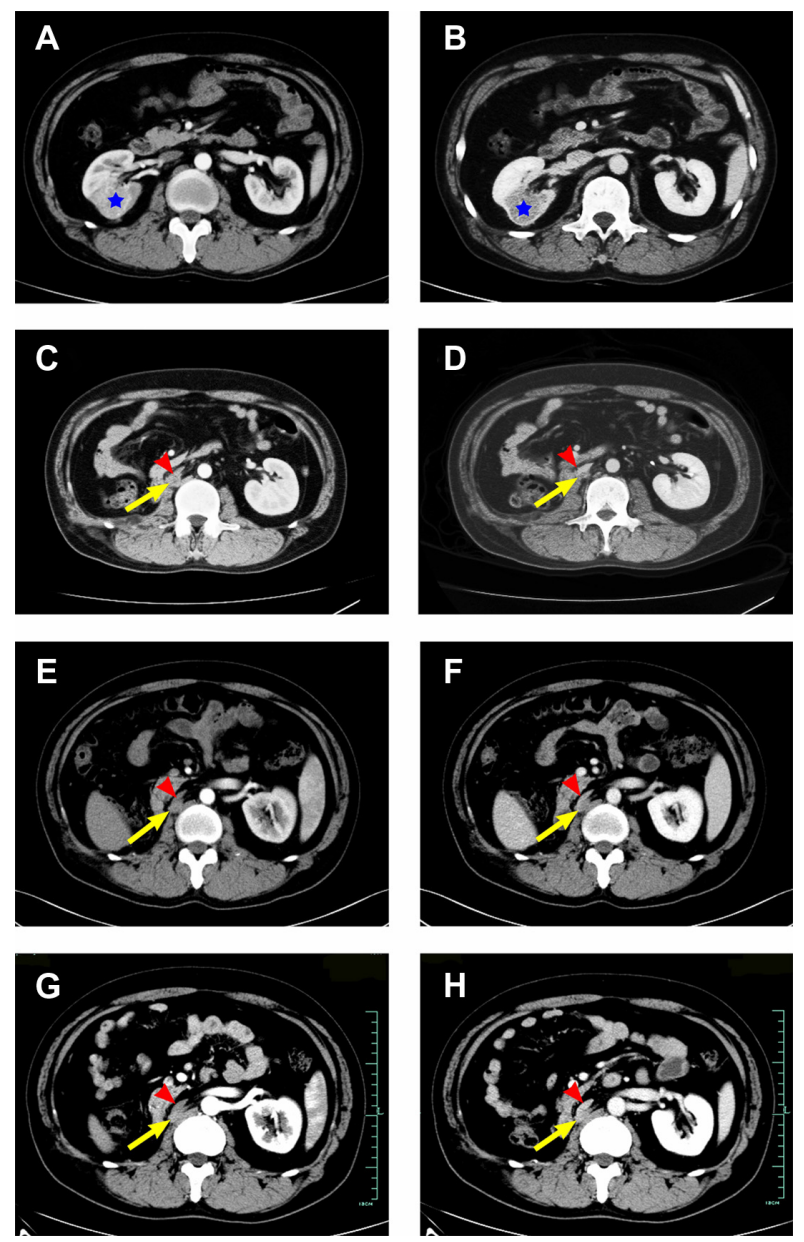

Figure I The consecutive abdominal CT scan images of the patient.

Notes: His first CT scan images taken 5 years ago identify the right renal tumor (enhanced [A], enhanced [B]); his CT images at regular re-examination postdischarge in 2010 (enhanced [C], enhanced [D]), 2012 (enhanced [E], enhanced [F]), and eventually 2013 (enhanced [G], enhanced [H]). Retrospectively, the granuloma appeared 6 months after the nephrectomy, and had not been identified during the follow-up. There is no significant development of the granuloma during the follow-up. The pentagram denotes the renal cancer, the yellow arrow indicates the granuloma, and the red arrow head indicates the inferior vena cava.

Abbreviation: CT, computerized tomography. annually (Figure 1). However, the 18F-fluorodeoxyglucose (FDG)-PET/CT scan detected a suspicious mono-focal lesion with significantly higher maximum standardized uptake value (SUVmax) than the surroundings, measuring $2.6 \times 3 \mathrm{~cm}$ with SUVmax: 9.85 (Figure 2). Retrospectively, the hidden lesion was pinpointed just behind the right edge of the inferior vena cava (IVC), adjacent to the right crus of the diaphragm on images 6 months after surgery; the ill-defined nodular lesion was slightly and homogeneously enhanced in contrast-material phase. The diagnosis was suspected to be lymph node metastasis of clear cell RCC. Needle aspiration biopsy guided by ultrasound localization was dismissed without hesitation, due to the difficulty in accessing the lesion surrounded by vital organs. No relapserelated syndrome, physical sign, or abnormal laboratorial tests were presented.

Investigational laparotomy was selected as the least risky invasive procedure. Intraoperatively, a fibrous mass measuring about $4 \mathrm{~cm}$ in major diameter was attached posterior to the IVC, under the right renal vein stump severed during the first operation. Unexpectedly, the mass was firmly attached and could not be clearly exposed in situ. The IVC was partially freed and then occluded at the upper level of the mass without hampering reflux of the left renal vein (Figure 3). The vital signs were reported to be within the normal range. Then, the

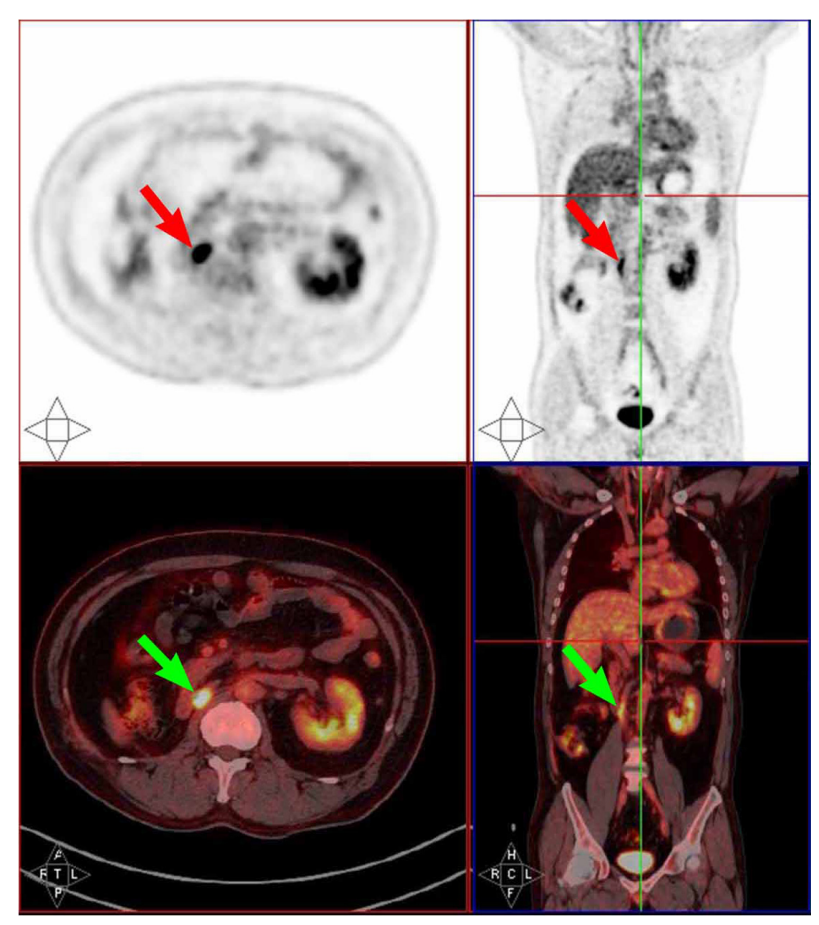

Figure 2 The 18F-fluorodeoxyglucose positron emission tomography scan of the patient in 2013.

Note: The arrow indicates the granuloma. 

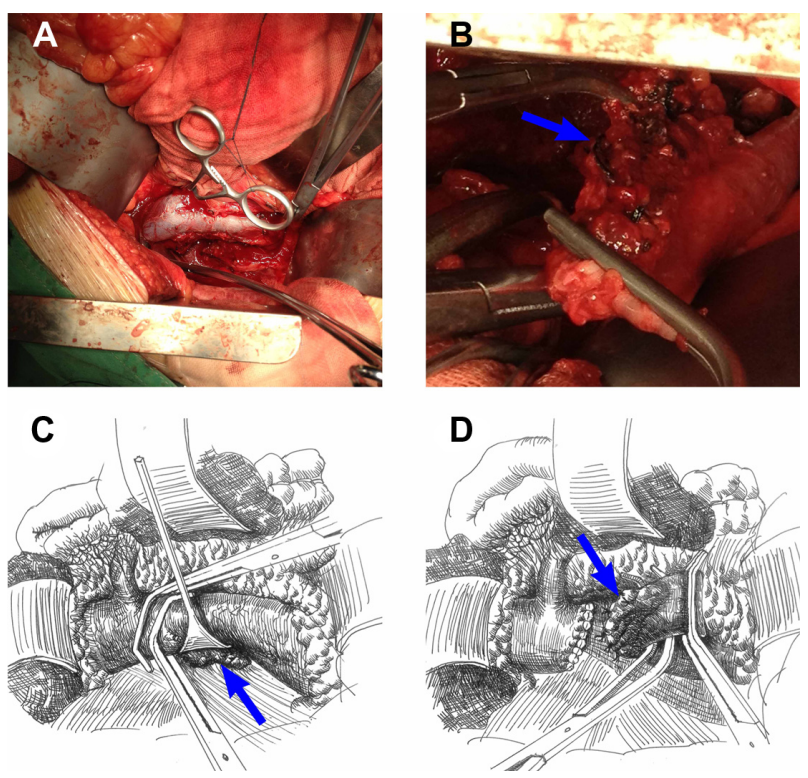

Figure 3 The intraoperative photographs and schematic renderings show details of the operation.

Notes: (A) A sizable segment of IVC around the lesion was freed and detached, where two Satinsky's clamps were placed at the proximal part to occlude the IVC without affecting the left renal vein; (B) the granuloma (blue arrow) was exposed and detached from posterior tissue after the proximal IVC segment was ligated, cut off, and stretched caudally. (C and D) The sketch illustrations explain the key steps straightforward. (C) The granuloma was attached posterior to the IVC (arrow). (D) The IVC was ligated and sutured after the granuloma was removed (arrow).

Abbreviation: IVC, inferior vena cava.

IVC was clamped, severed, and retracted to expose and dissect the lesion. Unfortunately, separation of the lesion tore the vessel wall, which caused bleeding. The surgeon occluded the IVC at lower level of the lesion, and severed it. The lesion was finally resected along with the IVC segment. The operation from clamp of vein to removal of the lesion lasted 35 minutes, during which the vital signs of the patient were basically stable (arterial blood pressure was $60-75 \mathrm{mmHg}$ for diastolic pressure, $80-100 \mathrm{mmHg}$ for systolic pressure; heart rate was around 70 beats per minute; central venous pressure ranged from 6-10 $\mathrm{cm} \mathrm{H}_{2} \mathrm{O}$ ).

Considering the sizable length of the defective vessel, it was impossible to perform end-to-end anastomosis. The polytetrafluorothylene vascular graft was recommended by the vascular surgeon to re-establish the caval continuity, but was denied by the patient's family out of economic consideration. The IVC pressure was assessed by pressure monitoring at the iliac vein and the reading was $25 \mathrm{~cm} \mathrm{H}_{2} \mathrm{O}$; it was suggested that ligating and suturing the stumps to simply close the IVC was an acceptable alternative.

The patient recovered well without complaints of extremity pain or swelling. No edema, stasis dermatitis, or skin ulcer was found after operation. The ultrasound scan confirmed no thrombosis in his deep veins of lower extremity.
Grossly, no visible "foreign body" was found in the sectioned specimen, except for pale fibrous tissue, while microscopically the lesion was diagnosed with a foreignbody granuloma embedded with surgical sutures and necrotic adipose tissue (Figure 4).

\section{Discussion}

Granulomatous reaction to a foreign body refers to an immunological response to the exogenous material such as surgical suture, gauze, talc, silicon, and fragment of a tile, or endogenous material like cholesterol. With the increasing use of novel surgical material, the incidence of granuloma formation due to iatrogenic factors is on the rise. Pseudo tumors due to sponge retention are reportedly estimated to occur in 1 in 1,000-18,000 procedures. ${ }^{4,5}$ In our case, the granuloma was confirmed to contain surgical suture used in the previous operation. During the nephrectomy, the surgeon encountered bleeding when he performed a lymphadenectomy; this accident was probably attributed to inadvertent injury of an aberrant lumbar vein. The surgeon controlled the bleeding point by figure-of-eight suturing using absorbable suture Dexon 4-0 (Tyco Healthcare UK Ltd, Gosport, UK). We surmised that it was this stitch that caused the granuloma.

In general, typical foreign-body granulomas are discernible by traditional diagnostic tools. On sonographic images, a granuloma may appear as a hypoechoic lesion with/without posterior acoustic shadowing, and without Doppler flow signals. ${ }^{6}$ On magnetic resonance imaging (MRI), it usually appears hyper-intense against a hypointense signal of the foreign body on T2-weighted images. ${ }^{7}$ On contrast-material CT images, the lesion may undergo rim enhancement, if the foreign body can be visualized. ${ }^{7,8} \mathrm{PET} /$ CT has been widely used for the diagnosis and evaluation of many cancers. Despite the long-debated efficaciousness of PET to distinguish the malignant from the benign due to the overlapping standardized uptake value of these lesions, foreign-body granulomas harboring foreign bodies may take on unique appearances on PET. Miyake et al has reported that some large granulomas with visible embedded foreign bodies (textile) might present themselves with a characteristic ring-shaped FDG uptake pattern, resembling an abscess or tumor with significant necrosis. ${ }^{9}$ Comparatively, atypical granulomas without visible foreign bodies may appear as dubious lesions by mimicking primary or metastasized malignancies or cancerous lymphadenopathies found incidentally by imaging. An incidental detection of such a suspected mass might very likely result in unnecessary surgery as described in our case. ${ }^{1,2}$ 

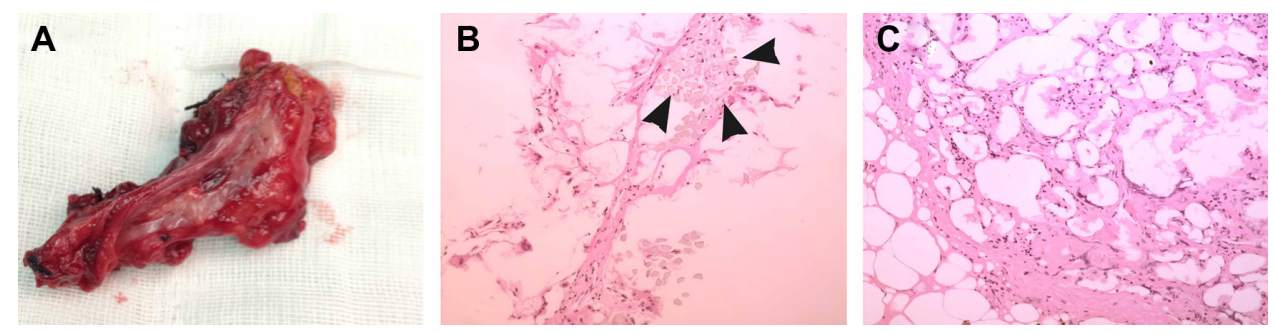

Figure 4 The photographs and microscopic pathological presentation of the surgical specimen.

Notes: (A) Grossly, the granuloma sized $2.6 \times 3 \mathrm{~cm}$ is covered by a fragment of inferior vena cava; (B) the arrowheads indicate the surgical suture; (C) numerous epithelioid histiocytes, multinucleated giant cells, and the amorphous necrotic fat are present.

In our case, the lesion was initially detected by PET/CT instead of contrast-enhanced CT. On PET, the equivocal mass is seemingly as tracer-avid as the parenchyma, and its SUVmax is within the domain of that of recurring RCC as shown by some studies, ${ }^{10,11}$ which is suggestive of tumor recurrence or metastasis. As for CT, although the insidious lesion in our case undergoes enhancement in a mild and homogeneous pattern, this mono-focal nodular mass with retrocaval position is uncommon for a suspicious lymph-metastasized RCC. Generally, aggressive clear cell RCCs that exhibit hyper-attenuation and central necrosis on images are prone to accompany lymph nodes metastasis; ${ }^{12}$ and the lymphadenopathy appears as multiple paracaval and/or para-aortic oval-shaped lesions with obliteration of surrounding fat planes on images. In addition, it should be noted that the nodular lesion initially appeared on CT images 6 months after the surgery and no change had been found during the course of 4 years until PET/CT was utilized. That is sharply different from a malignancy. Recurrent RCCs usually appear as lumps of hypodensities with necrosis and progressively local invasion; in contrast-enhanced phases, they tend to present with rapid and inhomogeneous enhancement. ${ }^{13}$ Therefore, the diagnosis of a malignant lesion is radiographically untenable.

Clinically, clear cell RCC represents as a highly metastasis-predisposing entity, accounting for more than $90 \%$ of all metastatic RCCs. In our case, the primary tumor extirpated in the first operation was a "small renal cancer tumor" (diameter $<4 \mathrm{~cm}$ ) without extrarenal invasion. It was classified as TNM (tumor, lymph node, metastasis) stage T1 and low grade nuclear grading (Fuhrman II grade), which indicated its low aggressiveness. ${ }^{14}$ Hence, the likelihood of postoperative metastasis or recurrence for such a lesion is theoretically low.

The foreign-body granulomas per se are generally asymptomatic and benign, and thus seldom need surgical intervention. The surgically treated tumors are either complication-causing lesions ${ }^{15}$ or cancer-mimicking ones. In addition, some granulomas were documented to be invasive, ${ }^{16,17}$ and this might account for the failed dissection of the lesion from the IVC in our case, which remarkably complicated the surgery.

As for the surgery detail, resection of the IVC is certainly not a preferential choice but an improvised solution. Although it is generally safe and feasible to close the IVC without significantly impairing the venous reflux, there are still some possible complications to be noted including edema, stasis dermatitis, and skin ulcers; these presentations originate from poor blood return to lower extremities. An extreme case that resulted in death was once reported, which was characterized by pelvic thrombosis 11 years after ligation of IVC. ${ }^{18}$ We surmise that the varying presence and robustness of collateral vessels might account for those complications. Currently, there is no conclusive standard on safe ligation of IVC as far as we know, but venous pressure more than $30 \mathrm{~cm} \mathrm{H}_{2} \mathrm{O}$ theoretically indicates vessel restoration. In our case, the pressure reading of the iliac vein was favorably $25 \mathrm{~cm} \mathrm{H}_{2} \mathrm{O}$ during the operation, which convinced us that the closure would be simple with minor likelihood of venous hypertension.

In summary, the diagnosis of a foreign-body granuloma could be so challenging that no single routine diagnostic modality is reliable. Invasive diagnosis by aspiration biopsy may be the most accurate method, but sometimes its use is limited by physical conditions, and is replaced with an exploratory surgery. Anyway, the diagnosis needs a more cautious and comprehensive approach; besides radiographic differentiation, critical analysis of symptoms, complications, and a thorough evaluation of medical history are of equal importance.

\section{Acknowledgments}

This study was supported by grants from the Scientific Research Fund of Zhejiang Provincial Education Department (Grant No Y201120149), the Scientific Research Fund of the Health Bureau of Zhejiang Province (Grant No 2013KYB099), and the Scientific Research Fund of the Science Technology 
Department of Zhejiang Province (Grant No 2012R10034). Special thanks should be given to Dr Michael Stanga, Washington State University, USA, who made the effort to polish and refine the written English.

\section{Author contributions}

Jindan Luo took part in the operation and edited the manuscript as a major contributor. Yeqing Mao took part in the operation, drafting the article, providing sketch illustrations, and language polishing. Songliang Cai took took part in the operation. Xiaoyong Shen took charge of radiological exams. Shanwen Chen took part in the operation, caring for the patient, and conceptualized the paper. Liping Xie conceptualized the paper and revised it critically. All authors contributed toward data analysis, drafting, and revising the manuscript.

\section{Disclosure}

The authors declare no conflicts of interest in this work.

\section{References}

1. Rehak Z, Szturz P, Krejci E, Kocakova I. FDG-PET-positive foreignbody granuloma mimicking residual germinal tumor infiltration. Clin Nucl Med. 2012;37(8):790-792.

2. Dogra PN, Tandon S, Ansari MS, Anupama, Chopra P. Suture foreign body granuloma masquerading as renal neoplasm. Int Urol Nephrol. 2005;37(1):27-29.

3. Tefik T, Sanli O, Oktar T, Yucel OB, Ozluk Y, Kilicaslan I. Oxidized regenerated cellulose granuloma mimicking recurrent mass lesion after laparoscopic nephron sparing surgery. Int J Surg Case Rep. 2012;3(6): 227-230.

4. Gawande AA, Studdert DM, Orav EJ, Brennan TA, Zinner MJ. Risk factors for retained instruments and sponges after surgery. $N$ Engl J Med. 2003;348(3):229-235.

5. Rappaport W, Haynes K. The retained surgical sponge following intra-abdominal surgery. A continuing problem. Arch Surg. 1990;125(3):405-407.
6. Kim EY, Ko EY, Han BK, et al. Sonography of axillary masses: what should be considered other than the lymph nodes? J Ultrasound Med. 2009;28(7):923-939.

7. Hashim H, Alli K, Faridah Y, Rahmat K. Cotton-ball granuloma mimicking axillary lymphadenopathy in a breast cancer patient. Biomed Imaging Interv J. 2011;7(3):e19.

8. Ando A, Hatori M, Hagiwara Y, Isefuku S, Itoi E. Imaging features of foreign body granuloma in the lower extremities mimicking a soft tissue neoplasm. Ups J Med Sci. 2009;114(1):46-51.

9. Miyake KK, Nakamoto Y, Mikami Y, et al. F-18 FDG PET of foreign body granuloma: pathologic correlation with imaging features in 3 cases. Clin Nucl Med. 2010;35(11):853-857.

10. Nakhoda Z, Torigian DA, Saboury B, Hofheinz F, Alavi A. Assessment of the diagnostic performance of (18)F-FDG-PET/CT for detection and characterization of solid renal malignancies. Hell J Nucl Med. 2013;16(1):19-24.

11. Khandani AH, Cowey CL, Moore DT, Gohil H, Rathmell WK. Primary renal cell carcinoma: relationship between $18 \mathrm{~F}-\mathrm{FDG}$ uptake and response to neoadjuvant sorafenib. Nucl Med Commun. 2012;33(9): 967-973.

12. Koh DM, Hughes M, Husband JE. Cross-sectional imaging of nodal metastases in the abdomen and pelvis. Abdom Imaging. 2006;31(6): 632-643.

13. Chae EJ, Kim JK, Kim SH, Bae SJ, Cho KS. Renal cell carcinoma: analysis of postoperative recurrence patterns. Radiology. 2005;234(1): 189-196.

14. Fuhrman SA, Lasky LC, Limas C. Prognostic significance of morphologic parameters in renal cell carcinoma. Am J Surg Pathol. 1982;6(7): 655-663.

15. Kyriakou G, Kalahanis N, Kyroudi-Voulgari A, Zervas A, Mitropoulos D Extravesical foreign body presenting as a bladder tumor. Urol Int. 2000;65(4):224-225.

16. Bella Z, Torkos A, Tiszlavicz L, Ivan L, Jori J. Cholesterol granuloma of the maxillary sinus resembling an invasive, destructive tumor. Eur Arch Otorhinolaryngol. 2005;262(7):531-533.

17. Murugasu E, Yong TT, Yoon CP. Invasive middle ear cholesterol granuloma involving the basal turn of the cochlea with profound sensorineural hearing loss. Otol Neurotol. 2004;25(3):231-235.

18. Le Lourd R, Le Lourd F, Beauvieux Y. [Ligation of the inferior vena cava between the 2 renal veins. Death by pelvic thrombosis 11 years later (general considerations)]. J Med Bord. 1961;138:613-627. French.
OncoTargets and Therapy

\section{Publish your work in this journal}

OncoTargets and Therapy is an international, peer-reviewed, open access journal focusing on the pathological basis of all cancers, potential targets for therapy and treatment protocols employed to improve the management of cancer patients. The journal also focuses on the impact of management programs and new therapeutic agents and protocols on

\section{Dovepress}

patient perspectives such as quality of life, adherence and satisfaction. The manuscript management system is completely online and includes a very quick and fair peer-review system, which is all easy to use. Visit http://www.dovepress.com/testimonials.php to read real quotes from published authors. 